\title{
Validation of the Couples Resource Map Scales
}

\author{
By: Christine E. Murray and Allison M. Forti
}

Murray, C.E. \& Forti, A.M. (2009). Validation of the couples resource map scales. Journal of Couple \& Relationship Therapy: Innovations in Clinical and Educational Interventions, 8(3), 209-225. doi: 10.1080/15332690903048812.

***Note: This version of the document is not the copy of record. Made available courtesy of Taylor \& Francis Group, LLC. Link to full text: http://dx.doi.org/10.1080/15332690903048812

\section{FIGURE 1 IS OMITTED FROM THIS FORMATTED DOCUMENT.}

\begin{abstract}
:
This article describes an examination of the concurrent and criterion-related validity of the Couples Resource Map Scales (CRMS), a 63-item assessment instrument designed to assess the levels of support available to couples from various personal, relationship, and contextual resources. The sample of 1,103 participants included representatives of a nonclinical population and clients in therapy. The results supported the validity of the CRMS (a) by demonstrating statistically significant relationships between the three Resource Area Scales and other measures of related constructs, (b) through a regression analysis linking the CRMS Resource Area Scales to relationship satisfaction, and (c) by examining the differences in scores between the clinical and nonclinical samples. Implications for using the CRMS with clients are outlined.
\end{abstract}

\section{Article:}

Strength-based approaches in the field of marriage and family therapy (MFT) assume that clients are able to reach their treatment goals when they identify and mobilize available and newly developed resources to address the challenges that bring them to therapy (Murray \& Murray, 2004; Walter \& Peller, 1992). For couples who present for therapy, relevant resources may include personal strengths, relationship resources, and support from the social context. Due to the potentially broad range of resources available to support couples, MFTs may overlook certain client resources if they do not assess specifically for them. By overlooking these resources, therapists and clients can miss important possible solutions to couples' presenting problems. Therefore, an assessment instrument designed to measure the degree of support that clients receive for their relationships from personal, relationship, and contextual resources is a useful tool for clinical practice. The present study details the steps taken to validate one such measure, the Couples Resource Map Scales (CRMS).

The CRMS (Murray, 2007) is a 63-item self-report assessment instrument that measures the support couples receive from various personal, relationship, and contextual resources. The CRMS was designed to be useful and accessible to both practitioners and researchers of couple therapy. The CRMS has the unique distinction of being available (free-of-charge) through an interactive website on the Internet (http://www.couplesresourcemap.org). Individuals who complete the online assessment receive immediate, printable feedback that depicts the amount of support available to their relationships from 21 resources organized in three broad categories of 
personal, relationship, and contextual resources. These three categories correspond to the instrument's three Resource Area Scales, which are the focus of the current investigation. Practicing MFTs can direct their clients to the assessment website and subsequently discuss the printed feedback with their clients. The CRMS, which is also usable in a paper-and-pencil format, also can be a valuable resource to MFT researchers who wish to study the associations among various resources and other aspects of relationship functioning (e.g., relationship satisfaction and stability, outcomes in couple therapy, and protective factors against relationship distress). The present study was designed to assess the concurrent and criterion-related validity of the instrument in its current format using a sample that included participants from nonclinical and clinical samples.

\section{REVIEW OF THE LITERATURE}

\section{Theoretical Foundation of the CRMS}

The field of MFT has demonstrated a gradual shift away from approaches that focus on pathology and dysfunction toward approaches that focus on resources and strengths (O'Connell, 1998). One important strength-based approach that emerged in recent years is Solution-Focused Therapy (SFT; Brief Family Therapy Center, 2007), which serves as the theoretical foundation for the CRMS. Several previous authors have applied the solution-focused approach to clinical work with couples (e.g., Hoyt \& Berg, 1998; Murray \& Murray, 2004). This theory holds that focusing on clients' strengths and resiliencies leads to positive changes (Murray \& Murray, 2004; Walter \& Peller, 1992). SFT shifts the focus of therapy away from explaining problems and instead highlights exceptions to the problem (i.e., times when the problem does not occur or occurs less) and solutions (i.e., what life will or does look like when the problem does not exist) (DeJong \& Berg, 1998; O’Connell, 1998). An important assumption of SFT is that clients have the ability to create their own solutions based on resources to which they already have access (Murray \& Murray, 2004; Walter \& Peller, 1992). Therefore, resources play an important role in SFT, in that they provide the foundation upon which clients build solutions as they work toward positive changes.

Consistent with the solution-focused assumption that clients build on their available resources to make desired changes, existing research supports the benefits that personal, relationship, and contextual resources provide to couples in their relationships. Personal resources are resources found within the individual. On the CRMS, these resources include self-awareness, self-soothing strategies, coping skills, personal dreams, values, and self-esteem. Personal resources have been linked to increased marital satisfaction and other positive relationship outcomes (e.g., Bodenmann \& Shantinath, 2004; Gottman, 1999). Relationship resources describe resources shared within a couple's relationship. The CRMS assesses the following relationship resources: relationship skills, strategies to manage negativity, knowledge about one's partner, shared material resources, shared dreams, and a shared history. Conflict resolution and communication skills are two relationship resources that prior research demonstrates are important for positive relationship health (Gottman, 1999; Holman et al., 2001). The third category of resources included on the CRMS is contextual resources, which describes resources that exist within the couple's social context. These include families-of-origin, friends, extended social networks, careers, the economic and political context, family life professionals, and cultural and community resources. Previous research demonstrates the important role these resources play in contributing to positive relationship qualities, particularly friendships and community support 
(Holman et. al, 2001) and family of-origin factors (Larson \& Holman, 1994). Interested readers should consult Murray (2007) for detailed descriptions of each resource.

\section{Prior Phases of Development of the CRMS}

This section presents a brief description of the initial steps taken previously to develop the instrument. The CRMS is based on the Couple's Resource Map (Figure 1), which appeared originally in the literature in Murray and Murray (2004). At the time, the map was intended to be a qualitative assessment instrument. The response to the map from the professional community demonstrated the high level of professional interest in a resource-based approach to assessment of couple relationships, in that approximately 175 to 200 professionals requested a copy of the map following publication of that article, with requests continuing to be made nearly 4 years following publication. Therefore, the map appeared to be a relevant conceptual framework for developing a standardized, empirically based instrument to provide professionals with an alternative strategy for assessing couples’ relationship resources.

Three phases of instrument development were then completed (Murray, 2007). The first involved the development of the item pool and an expert panel review to establish the face validity of the instrument. The second phase examined the psychometric properties of the instrument. This phase involved a survey of 397 university seniors who were involved in intimate monogamous relationships. The initial analyses demonstrated strong internal consistency reliability for the total scale and three Resource Area Scales (Personal, Relationship, and Contextual). The third phase provided a preliminary examination of the validity of the CRMS by testing the relationship between CRMS total and subscale scores and relationship satisfaction. The results demonstrated a general trend for participants with higher CRMS total and subscale scores to also demonstrate higher levels of relationship satisfaction.

Following the previous work described in this section, the present study aimed to examine the concurrent and criterion-related validity of the CRMS using a larger sample, including participants who represent both clinical and nonclinical populations. The following research questions guided this study: (a) What is the relationship between participants' CRMS Resource Area Scale scores and their scores on other existing measures that assess related constructs (i.e., self-esteem, relationship satisfaction, and social support)? (b) Can participants' CRMS Resource Area Scale scores be used to predict their levels of relationship satisfaction? (c) Are there differences in participants' CRMS total and Resource Area Scale scores based on whether they are members of the clinical or nonclinical sample?

\section{METHOD \\ Instrumentation}

This study involved an anonymous Internet-based survey. The instrumentation was available to participants through an Internet-based website devoted exclusively to the CRMS. The interactive Internet site scored participants' CRMS responses and provided immediate, printable colorcoded feedback in the form of a diagram depicting the resources available to support participants' relationships. This feedback was presented to participants and counselors as an incentive for participating in the study. The Internet-based format was designed to render the CRMS to be useful and accessible to both MFT researchers and practitioners. 
The instrumentation included a demographic questionnaire to assess the background characteristics of the participants and their relationships, the CRMS (Murray, 2007), the Rosenberg Self-Esteem Scale (RSES; Rosenberg, 1989), the Relationship Assessment Scale (RAS; Hendrick, 1988), and the Provision of Social Relations (PSR; Turner, Frankel, \& Levin, 1983). Although each of the Resource Area Scales is multidimensional (i.e., each encompasses a number of resources that fall within the sphere), we selected one related construct per sphere to provide an economical, illustrative measure of concurrent validity for each scale. The constructs of self-esteem, relationship satisfaction, and social support were selected because they were deemed to be generally representative and to encompass the specific resources included in the corresponding resource spheres.

Demographic Questionnaire: The demographic questionnaire measured the following participant characteristics: participant's gender, age, ethnic background, level of education, and annual income; the length of the couple's relationship; the number of children between the partners; whether the couple cohabits; relationship status; current participation in couple or individual counseling or therapy; and city and state of residence.

The Couples Resource Map Scales: The CRMS (Murray, 2007) asks participants to indicate the extent to which they agree or disagree with 63 statements measuring the amount of support they receive for their relationships from various personal, relationship, and contextual resources. Participants respond using a Likert scale with responses ranging from 1 (strongly disagree) to 4 (strongly agree). For all scales, higher scores indicate higher levels of available resources. The CRMS is designed for three levels of assessment. The total scale, including all 63 items, assesses the overall level of support available to the respondent's relationship. In the previous study of the CRMS, this scale demonstrated an internal consistency reliability (Cronbach's alpha $[\alpha]$ coefficient) of .94. The next level of scales includes the three Resource Area Scales. The previous study demonstrated the following internal consistency reliability coefficients for these scales: Personal Resource Area Scale (PRAS; $\alpha=.88$ ), Relationship Resource Area Scale (RRAS; $\alpha=.92$ ), and Contextual Resource Area Scale (CRAS; $\alpha=.86$ ). The third level involves the 21 subscales (with three items each) assessing the specific categories of resources that fall under the Resource Area Scales. These 21 subscales relate to the small circles on the Couple's Resource Map (Figure 1). The average internal consistency reliability coefficient for these scales in the previous study was $\alpha=.72$, with coefficients for the individual scales ranging from $\alpha=.40$ to $\alpha=.88$. In the current study, the focus of the analyses was on the total scale and the three Resource Area Scales, and the 21 subscales were not examined. [See Murray (2007) for a list of the items included on the CRMS.]

Rosenberg Self-Esteem Scale: The RSES includes 10 items that measure respondents' overall sense of self-worth (Rosenberg, 1989; The Morris Rosenberg Foundation, 2006). Higher scores indicate more positive self-esteem; lower scores indicate more negative self-esteem. The RSES demonstrates good internal consistency reliability, with coefficients ranging between $\alpha=.77$ and $\alpha=.88$ across a number of studies (The Morris Rosenberg Foundation, 2006).

Relationship Assessment Scale: The RAS is a seven-item instrument that assesses a respondent's general satisfaction with his or her intimate relationship (Hendrick, 1988). Higher scores indicate more positive relationship satisfaction. Hendrick demonstrated that the RAS 
measures a single factor and has an internal consistency reliability coefficient of $\alpha=.86$. The validity of the RAS was established by demonstrating relationships in the expected directions between the instrument and alternative measures of relationship satisfaction.

Provision of Social Relations: The PSR is a 15-item instrument designed to assess the level of social support that the respondent perceives to be available to him or her (Turner et al., 1983). In this study, the respondents' PSR scores were coded so that higher scores on the PSR indicate higher levels of social support. The PSR demonstrates good internal consistency reliability (with demonstrated internal consistency reliability coefficients ranging from $\alpha=.75$ to $\alpha=.87$ ) and concurrent validity (Corcoran \& Fischer, 2000b).

\section{Sample Recruitment Strategies}

The sample was comprised of both clinical and nonclinical participants. To be eligible to participate in this study, participants must have met the following two eligibility criteria: (a) participants must have been at least 18 years of age and (b) participants must have been involved in a current monogamous intimate relationship. For purposes of this study, monogamous intimate relationship was defined as an exclusive relationship in which two individuals share an emotional, romantic, and/or sexual connection and both individuals agree that neither partner will share a similar relationship with another person (Murray, 2007). Individuals in heterosexual and same-sex relationships were eligible to participate. Participants were recruited individually and were therefore not required to participate with their partners. Because the survey website was anonymous, no efforts were made to determine whether any participants were partners within the same relationship.

The nonclinical sample was recruited by sending e-mails to all juniors, seniors, and graduate students who were enrolled in a mid-size public university in the southeastern United States during the Fall 2006 semester. In addition to the nonclinical sample, a clinical sample was sought. The clinical sample included individuals who were receiving current individual or couple therapy focusing on relationship issues. The clinical sample was recruited through contacts with the clinicians who provide these services. These clinicians include professional counselors, marriage and family therapists, psychologists, clinical social workers, and pastoral counselors. We used three strategies for contacting clinicians to request that they invite their clients who were seeking services related to relationship issues to participate in the study. First, we made contacts with clinicians through a presentation of previous work on the CRMS at a professional conference. Second, approximately 20 clinicians contacted the first author requesting a reproducible version of the Couple's Resource Map [as was offered in Murray and Murray (2004)] during the data collection period. In the e-mail in which the maps were provided, these individuals were provided with a brief description of the current study and a link to the study website in the event that they wished to invite their clients to participate. Third, we made personal contacts (via postal mail) with local and regional clinicians to request that they invite their clients to participate in the study. We located clinicians through an Internet search of an online directory of mental health professionals (www.therapistlocator.net, hosted by AAMFT), as well as through searches of local telephone directories. Clinicians who were contacted were provided with an informational brochure and postcards to distribute to their clients describing the study. 


\section{Data Collection Procedures}

This study was approved by the host university's Institutional Review Board prior to data collection. Data collection occurred via the Internet between September 1, 2006, and April 30, 2007. Due to the anonymous format of the survey and the various procedures used to recruit participants, it was not possible to calculate an accurate response rate, and the sample should therefore be considered one of convenience. Data collection for the nonclinical sample occurred in three phases spread throughout the data collection period. For the clinical sample, data collection occurred continuously throughout the entire data collection period.

\section{RESULTS}

\section{Description of the Sample}

A total of 1,103 participants comprised the final sample. The majority of respondents were female $(n=884,80.1 \%)$. The sample was predominantly white $(n=907,82.2 \%)$, followed by African American ( $n=105,9.5 \%)$, Asian American $(n=36,3.3 \%)$, other ethnic backgrounds $(n$ $=31,2.8 \%)$, Hispanic $(n=16,1.5 \%)$, and Native American $(n=7,0.6 \%)$. Participants reported an average age of 27.8 years ( $\mathrm{SD}=8.5$ years). The vast majority $(n=1,058,95.9 \%)$ of the respondents indicated that they resided in North Carolina (the state of the study's host university), although 14 other states and 3 other countries were represented by at least one participant. The participants' personal income levels were as follows: $\$ 15,000$ or less $(n=641$, $58.1 \%), \$ 16,000$ to $\$ 25,000$ ( $n=137,12.4 \%), \$ 26,000$ to $\$ 35,000(n=106,9.6 \%), \$ 36,000$ to $\$ 45,000(n=69,6.3 \%)$, and greater than $\$ 45,000(n=136,12.3 \%)$. The participants' highest levels of education completed were as follows: did not complete high school ( $n=1,0.1 \%)$, high school diploma ( $n=32,2.9 \%)$, some college but no degree $(n=333,30.2 \%)$, associate's degree ( $n=83,7.5 \%)$, bachelor's degree $(n=193,17.5 \%)$, some graduate school $(n=295,26.7 \%)$, master's degree $(n=148,13.4 \%)$, and doctoral degree $(n=14,1.3 \%)$.

Most participants ( $n=1,040,94.2 \%$ ) indicated that they were involved in heterosexual intimate relationships, while 63 participants (5.7\%) indicated that they were involved in same-sex relationships. The average length of their intimate relationships was 5.6 years $(\mathrm{SD}=6.3$ years), with a range from less than 1 year to 35 years. Two hundred eighty (25.3\%) participants reported that they and their partners had any children (together or from previous relationships), with the number of children ranging from 1 to 7 . The participants represented a variety of relationship statuses, with 475 (43.1\%) participants indicating that they were dating, $126(11.4 \%)$ indicating that they were engaged, 375 (34.0\%) indicating that they were married, and $126(11.4 \%)$ indicating that they were living with their partners but were unmarried. Of the total sample, 582 (52.8\%) participants reported living in the same households as their partners.

Questions on the demographic questionnaire were used to differentiate the clinical sample (i.e., participants reporting that they were participating currently in individual or couple therapy) from the nonclinical sample (i.e., participants reporting no current participation in individual or couple therapy). The clinical sample included 119 participants, representing $10.8 \%$ of the total sample; the nonclinical sample included 984 participants, representing $89.2 \%$ of the total sample. Oneway ANOVAs and chi-square tests were conducted to determine whether there were any significant differences in the demographic characteristics of the nonclinical and clinical samples. There were no significant differences between the clinical and nonclinical samples based on the participants’ ages or ethnic backgrounds; length of relationship; gender; and whether the 
participants reported having any children. However, there were small but significant differences between the nonclinical and clinical samples based on relationship status $\left(\chi^{2}=7.884, d f=3, p=\right.$ $.05)$ and participants' education levels $\left(\chi^{2}=15.518, d f=8, p=.05\right)$. Compared with the nonclinical sample, the clinical sample was composed of higher percentages of dating (46.2\% vs. 42.7\%) and engaged (17.6\% vs. 10.7\%) participants, but lower percentages of married (25.2\% vs. $35.1 \%)$ or living together (10.9\% vs. $11.5 \%)$ participants. Regarding highest levels of completed education, the clinical sample was comprised of lower percentages of participants who either had not completed high school or had a high school diploma $(0.8 \%$ vs. $3.3 \%)$ and participants with education beyond a bachelor's degree (37.8\% vs. $41.8 \%$ ), but higher percentages of participants with some college or an associate's degree (40.4\% vs. $37.4 \%)$ and participants with bachelor's degrees (20.2\% vs. 17.2\%).

\section{Internal Consistency Reliability of the CRMS}

Cronbach's alpha coefficients were calculated for the CRMS total scale and each of the Resource Area Scales. All of these scales demonstrated good internal consistency reliability, with the following results for each scale: CRMS total scale $(\alpha=.93)$, PRAS $(\alpha=.85)$, RRAS $(\alpha=.90)$, and CRAS $(\alpha=.84)$.

\section{Concurrent Validity of the CRMS Resource Area Scales}

Correlation analyses were used to determine the relationships between participants' CRMS (total and Resource Area Scale) scores and their scores on the measures assessing the related constructs of self-esteem, relationship satisfaction, and social support (Clark \& Watson, 1995). To support the concurrent validity of the Resource Area Scales, the three Resource Area Scales should be related most closely to the construct that corresponds to the level of assessment (i.e., personal, relationship, or social context). Table 1 depicts the correlation matrix for this analysis. The results of this analysis demonstrate statistically significant correlations between the CRMS Resource Area Scales and the corresponding measures of related constructs as expected. Moreover, the correlations between each Resource Area Scale and its corresponding measure were higher than the correlations between the other CRMS scales and those measures. Therefore, these results demonstrate support for the concurrent validity of the CRMS.

Table 1 Correlation Matrix for the CRMS and Measures of Related Construct

\begin{tabular}{llll}
\hline & RSES & RAS & PSR \\
\hline PRAS & $\mathbf{0 . 6 4 4 *}$ & $0.523^{*}$ & $0.381^{*}$ \\
RRAS & $0.322^{*}$ & $\mathbf{0 . 7 2 2}^{*}$ & $0.273^{*}$ \\
CRAS & $0.362^{*}$ & $0.452^{*}$ & $\mathbf{0 . 5 0 1}^{*}$ \\
CRMS Total & $0.491^{*}$ & $0.648^{*}$ & $0.463^{*}$ \\
\hline
\end{tabular}

Notes. Figures in bold represent the correlations between the CRMS Resource Area Scales and corresponding related construct.

*All correlations on this table are significant at the $p<.001$ level (two-tailed).

PRAS, CRMS Personal Resource Area Scale; RRAS, CRMS Relationship Resource Area

Scale; CRAS, CRMS Contextual Resource Area Scale; CRMS Total, Total scale score for the Couples Resource Map Scales; RSES, Rosenberg Self-Esteem Scale; RAS, Relationship Assessment Scale; PSR, Provision of Social Relations. 


\section{Prediction of Relationship Satisfaction Based on CRMS Scale Scores}

As a means of assessing the criterion-related validity of the CRMS scales, a regression analysis was conducted to determine whether participants' relationship satisfaction (as measured by the RAS) could be predicted by the availability of personal, relationship, and contextual resources (as measured by the PRAS, RRAS, and CRAS). The model tested included RAS scores as the dependent variable and PRAS, RRAS, and CRAS scores as the independent variables. The overall model was shown to be statistically significant $(F=406.97, d f=3, p<.001)$, and it explained $52.5 \%$ of the variance in participants' RAS scores (adjusted $R^{2}=0.525$ ). However, only PRAS $(\beta=.067, t=2.272, p=.02)$ and RRAS scale scores $(\beta=.657, t=22.583, p>.001)$ were significant predictor variables; CRAS scale scores $(\beta=.038, t=1.411, p=.16)$ did not account for any unique variance in addition to the variance accounted for by the PRAS and RRAS.

\section{Differences in CRMS Scores: Clinical and Nonclinical Samples}

An examination of the differences in CRMS scores between the clinical and nonclinical samples provided another approach to examining the criterion-related validity of the CRMS. We hypothesized that participants' CRMS scores (total scale and Resource Area Scales) would differ based on whether they were participating currently in therapy, with participants in the clinical sample demonstrating significantly lower scores on each scale. Members of the clinical sample (i.e., clients in therapy focusing on relationship issues) were likely to be experiencing relationship difficulties that prompted them to seek help, leading to an assumption that they would report fewer resources available to them across the various categories.

Table 2 presents the means for each sample on each scale. For all scales, the nonclinical sample demonstrated slightly higher mean scores, indicating higher levels of support from those resources. Our hypothesis was tested using a one-way ANOVA, with therapy participation (i.e., the clinical sample vs. the nonclinical sample) as the independent variable and CRMS total scale

Table 2 Clinical, Nonclinical, and Total Sample Means on the CRMS

\begin{tabular}{|c|c|c|c|c|}
\hline & PRAS & RRAS & CRAS & $\begin{array}{c}\text { CRMS } \\
\text { Total }\end{array}$ \\
\hline \multicolumn{5}{|c|}{ Nonclinical sample $(n=984)$} \\
\hline M & 58.70 & 57.76 & 76.76 & 193.22 \\
\hline SD & 6.04 & 7.52 & 10.32 & 20.52 \\
\hline \multicolumn{5}{|c|}{ Clinical sample $(n=119)$} \\
\hline M & 55.87 & 55.32 & 76.45 & 187.63 \\
\hline SD & 7.01 & 9.24 & 11.13 & 23.55 \\
\hline \multicolumn{5}{|c|}{ Total sample $(n=1103)$} \\
\hline M & 58.40 & 57.50 & 76.73 & 192.62 \\
\hline SD & 6.21 & 7.75 & 10.41 & 20.93 \\
\hline
\end{tabular}

Notes. PRAS, CRMS Personal Resource Area Scale; RRAS, CRMS Relationship Resource Area Scale; CRAS, CRMS Contextual Resource Area Scale; CRMS Total, Total scale score for the Couples Resource Map Scales. Possible ranges for each scale are as follows: PRAS, 18 to 72; RRAS, 18 to 72; CRAS, 27 to 108; CRMS Total, 63 to 252. 
and Personal, Relationship, and Contextual Resource Area Scale scores as the dependent variables. There were significant differences between the clinical and nonclinical samples on the PRAS $(F=22.58, d f=1, p<.01)$, RRAS $(F=10.60, d f=1, p=.001)$, and CRMS total scale $(F$ $=7.62, d f=1, p=.006)$. The difference between the groups on the CRAS was not significant ( $F$ $=0.10, d f=1, p=.76)$. Effect sizes were calculated to assist in the interpretation of these differences. There were small effect sizes for the differences between the groups on the PRAS $\left(R^{2}=0.02\right)$, RRAS $\left(R^{2}=0.01\right)$, and CRMS total scale $\left(R^{2}=0.01\right)$, but there was no effect determined for CRAS scores $\left(R^{2}=0.00008\right)$ (Penfield, 2003). As stated in the description of the sample section, there were differences between the clinical and nonclinical samples based on education and relationship status. A multivariate ANOVA was conducted that included education and relationship status as covariates. This analysis revealed no significant differences in participants' CRMS and Resource Area Scale scores based on education (PRAS: $F=3.22, d f=$ $1, p=.07$; RRAS: $F=0.02, d f=1, p=.89$; CRAS: $F=0.05, d f=1, p=.82$; CRMS total: $F=$ $0.13, d f=1, p=.72$ ) or relationship status (PRAS: $F=0.64, d f=1, p=.42$; RRAS: $F=2.15, d f$ $=1, p=.14$; CRAS: $F=0.93, d f=1, p=.34$; CRMS total: $F=0.09, d f=1, p=.77)$.

\section{DISCUSSION}

Together with the previous steps taken to develop the CRMS (Murray, 2007), the findings of this study provide evidence of the reliability and validity of the instrument and its three Resource Area Scales. Across two studies with different samples, the CRMS total scale and the Resource Area Scales have demonstrated strong internal consistency reliability coefficients, all falling within the desirable range of being above $\alpha=.80$ (Corcoran $\&$ Fischer, 2000a).

The concurrent validity of the Resource Area Scales was examined by analyzing the relationships between each scale and measures of related constructs for each level of assessment. Although all correlations included in this analysis proved to be statistically significant, the strongest relationships were found between each Resource Area Scale and its corresponding measure: the Personal scale was related most closely to the self-esteem measure, the Relationship scale was related most closely to the relationship satisfaction measure, and the Contextual scale was related most closely to the social support measure. This finding supports the concurrent validity of the grouping of relationship resources into these three categories. A future study involving a confirmatory factor analysis of the CRMS is needed in order to examine further this proposed framework for understanding the role of resources in couple relationships (Floyd \& Widaman, 1995).

This study included two means of examining the criterion-related validity of the CRMS. First, a regression analysis was used to determine whether participants' relationship satisfaction levels could be predicted by the CRMS Resource Area Scale scores. The model did, to a statistically significant level, explain a large proportion (52.5\%) of the variance in participants' relationship satisfaction. However, only the Personal and Relationship Resource Area Scales were significant predictor variables. Second, the CRMS scores of the clinical and nonclinical samples were compared to determine whether significant differences existed between the two groups. As predicted, the clinical sample demonstrated slightly lower average scores on the CRMS and the Resource Area Scales. The differences between the groups were statistically significant for all scales except for the CRAS, although the effect sizes of these differences was small, so the 
clinical significance of the differences may be minimal. Therefore, these final two analyses demonstrated further support for the validity of the CRMS, although these analyses suggest that additional research is needed to understand more fully the role that contextual resources play in supporting couples' relationship.

\section{Limitations and Directions for Future Research}

This study demonstrated limitations related to the sample and data collection procedures. The final sample should be considered a convenience sample, and the clinical sample was substantially smaller than the nonclinical sample. Because data was collected anonymously and on an individual level, it was not possible to determine whether partners from the same relationships completed the survey. Partners from within the same relationship may report similar relationship resources, and therefore would potentially have similar CRMS scores. Therefore, partners' scores could have overrepresented their relationship characteristics in the sample. Due to the large sample size, the impact of this limitation is likely to be minimal, although it would have had a greater potential influence on the clinical sample due to its smaller size. Regarding data collection, the survey did not assess the manner in which participants learned about the study. It is therefore possible that individuals who were not intended to be part of the sample were included (e.g., if an individual recruited into the nonclinical sample asked his/her partner who was not recruited to complete the survey to receive the printable feedback about their relationship). Similarly, it is possible that some of the participants who were counted as part of the clinical sample were actually recruited as part of the nonclinical sample (i.e., he or she was a university student who was also receiving therapy at the time of the study).

The current study provides early support for the applicability of the CRMS with clients in therapy. However, due to the relatively smaller size of the clinical sample included in this study, additional research is needed to study the use of the instrument with clinical samples. The focus of this study was on the three Resource Area Scales, but future research should also examine further the reliability and validity of the 21 three-item scales assessing the specific resources. Additional research also is needed to determine the test-retest reliability of the instrument, which will be useful in determining whether the CRMS is an appropriate outcome measurement for progress in therapy. Other clinically relevant research may involve (a) studying clients' reactions to receiving the CRMS feedback (e.g., Is the feedback encouraging or discouraging? Does the feedback make it more likely that clients will seek or remain in therapy?), (b) determining how closely CRMS scores are linked between relationship partners, (c) comparing CRMS scores based on clients' demographic backgrounds, (d) examining effective strategies for incorporating the instrument into therapy, and (e) determining if there are other resources (e.g., spirituality, sexuality, commitment, forgiveness, and kindness) that should be added to the instrument.

Future research is needed to look more closely at the role of contextual resources within couple relationships. Previous studies indicate contextual resources are important in determining relationship outcomes (Holman et al., 2001; Larson \& Holman, 1994). However, in the regression model in the current study, contextual resources did not account for any unique variance in relationship satisfaction, nor were there significant differences in contextual resources based on therapy participation. In contrast, personal and relationship resources were predictors of relationship satisfaction, and members of the nonclinical sample demonstrated slightly higher levels of personal and relationship resources compared to members of the clinical 
sample. Therefore, it appears possible that contextual resources are less influential on couples' relationships than other types of resources. Subsequent research is needed to examine the potential reasons for and consequences of this dynamic. Finally, another significant area for future research involves a confirmatory factor analysis to determine whether the underlying factor structure of the instrument matches the proposed framework of the CRMS (Floyd \& Widaman, 1995).

\section{Implications for Marriage and Family Therapy Practice}

The CRMS provides clinicians with a quick, useful means of assessing a wide range of personal, relationship, and contextual resources available to help support their clients in their relationships. The CRMS is designed to be a clinically relevant assessment device, and its Internet-based format renders it to be accessible to practitioners in a wide range of settings. The interactive design of the survey website offers immediate feedback to clients who complete the CRMS, in the form of a completed Couple's Resource Map that depicts the levels of support they receive from various resources. The printable feedback clients receive upon completion of the CRMS, therefore, becomes a useful tool for therapists in their clinical practice. Therapists can subsequently discuss this feedback with their clients during session, focusing on the application of existing resources to the clients' presenting problems. In addition, the completed Couple's Resource Maps can identify potential resources that could be strengthened to provide new sources of support for the clients.

As stated previously, the CRMS encompasses three levels of assessment of couple's relationship resources: an overall level of support, three broad categories of resources (personal, relationship, and contextual), and 21 specific resources within those broad categories. This study focused on the validity of the three Resource Area Scales, and therefore the clinical interpretation of the instrument is currently strongest at this level. However, the feedback provided to clients who complete the instrument will be most detailed at the level of the 21 specific resources (i.e., each resource will be encircled in a specific color to indicate the level of support received from it). Therefore, while interpreting clients' CRMS feedback, clinicians should look first at the general patterns that emerge in the Contextual, Relationship, and Personal Spheres, and then the specific levels of support indicated for each resource. Consider, for example, a client's map on which the color red (indicating a lot of support) encircles five out of six resources in the Personal Sphere, but the color yellow (indicating a little support) encircles six out of nine resources in the Contextual Sphere. This client's map could be interpreted as indicating that the support the client receives for his or her relationship is high from personal resources but minimal from contextual resources. Once the general patterns of support for a client's relationship have been identified, a more detailed discussion of the availability and application of the specific resources is warranted.

In their original presentation of the Couple's Resource Map as a qualitative assessment device, Murray and Murray (2004) provided the following suggestions for processing completed maps with clients. First, when both partners in a couple complete the survey, their maps can be compared for similarities and differences. Discussion of differences that exist between the partners' maps can lead to increased awareness of each partner's perspective about their relationship. Second, the therapist can focus the conversation on the areas in which the strongest levels of support are identified. These areas become clues about possible solutions to current problems within the relationship. Third, the therapist may work with clients to develop specific 
strategies for gaining greater support from resources from which low levels of support are indicated. These suggestions are as relevant for maps completed through the survey website as they are for maps completed through the qualitative procedures described originally.

The CRMS is useful for assisting clients in identifying potentially supportive resources. In the interpretation of the feedback, clinicians are responsible for assisting clients in determining how best to actually apply the identified resources to the clients' presenting problems. Within SFT, clinicians work with clients to co-construct solutions to presenting problems and promote progress in therapy (Friedman \& Lipchik, 1999). The solution-focused therapist assumes a nonexpert stance (DeJong \& Berg, 1998; O’Connell, 1998; Walter \& Peller, 1992), and therefore the clinician should assume that the client will be the best judge of how well an identified resource may apply to a particular presenting problem. Overall, we recommend that the CRMS be used primarily as a starting point for therapeutic conversation rather than as a diagnostic instrument.

\section{REFERENCES}

Bodenmann, G., \& Shantinath, S. (2004). The couples coping enhancement training (CCET): A new approach to prevention of marital distress based upon stress and coping. Family Relations, 53, 477-484.

Brief Family Therapy Center. (2007). What is solution-focused brief therapy? Retrieved July 12, 2007, from http://www.brief-therapy.org/

Clark, L. A., \& Watson, D. (1995). Constructing validity: Basic issues in objective scale development. Psychological Assessment, 7, 309-319.

Corcoran, K., \& Fischer, J. (2000a). Measures for clinical practice: A sourcebook. Volume 1: Couples, families, and children. New York: The Free Press.

Corcoran, K., \& Fischer, J. (2000b). Measures for clinical practice: A sourcebook. Volume 2: Adults. New York: The Free Press.

DeJong, P., \& Berg, I. K. (1998). Interviewing for solutions. Pacific Grove, CA: Brooks/Cole.

Floyd, F. J., \& Widaman, K. F. (1995). Factor analysis in the development and refinement of clinical assessment instruments. Psychological Assessment, 7, 286-299.

Friedman, S., \& Lipchik, E. (1999). A time-effective, solution-focused approach to couple therapy. In Donovan, J.M. (Ed.), Short-term couple therapy (pp. 325-359). New York: Guilford Press.

Gottman, J. M. (1999). The marriage clinic: A scientifically based marital therapy. New York: W. W. Norton.

Hendrick, S. S. (1988). A generic measure of relationship satisfaction. Journal of Marriage and the Family, 50, 93-98. 
Holman, T. B., Birch, P. J., Carroll, J. S., Doxey, C., Larson, J. H., \& Linford, S. T. (2001). Premarital prediction of marital quality or breakup: Research, theory, and practice. New York: Kluwer Academic/Plenum Publishers.

Hoyt, M. F., \& Berg, I. K. (1998). Solution focused couple therapy: Helping clients construct self-fulfilling realities. In F.M. Dattilio (Ed.) Case studies in couple and family therapy: Systemic and cognitive perspectives (pp. 203-232). New York: Guilford.

Larson, J. H., \& Holman, T. B. (1994). Premarital predictors of marital quality and stability. Family Relations, 43, 228-238.

Murray, C. E. (2007). Development of the Couples Resource Map Scales. Journal of Couple and Relationship Therapy, 6(4), 49-70.

Murray, C. E., \& Murray, T. L. (2004). Solution-focused premarital counseling: Helping couples build a vision for their marriage. Journal of Marital and Family Therapy, 30(3), 349358.

O’Connell, B. (1998). Solution-focused therapy. London: SAGE.

Rosenberg, M. (1989). Society and the adolescent self-image (rev. ed.). Middletown, CT: Wesleyan University Press.

The Morris Rosenberg Foundation. (2006). The Rosenberg Self-Esteem Scale. Retrieved May 28, 2007, from http://www.bsos.umd.edu/socy/grad/socpsy_rosenberg.html

Turner, R. J., Frankel, B. G., \& Levin, D. M. (1983). Social support: Conceptualization, measurement, and implications for mental health. Research in Community and Mental Health, 3, 67-111.

Walter, J. L., \& Peller, J. E. (1992). Becoming solution-focused in brief therapy. New York: Brunner/Mazel. 